\title{
Can Coupled Dark Energy Speed Up the Bullet Cluster?
}

\author{
Jounghun Lee ${ }^{1}$, Marco Baldi ${ }^{2,3}$
}

\begin{abstract}
It has been recently shown that the observed morphological properties of the Bullet Cluster can be accurately reproduced in hydrodynamical simulations only when the infall pairwise velocity $V_{c}$ of the system exceeds $3000 \mathrm{~km} / \mathrm{s}$ (or at least possibly $2500 \mathrm{~km} / \mathrm{s}$ ) at the pair separation of $2 R_{\text {vir }}$, where $R_{\text {vir }}$ is the virial radius of the main cluster, and that the probability of finding such a bullet-like system is extremely low in the standard $\Lambda \mathrm{CDM}$ cosmology. We suggest here the fifthforce mediated by a coupled Dark Energy (cDE) as a possible velocity-enhancing mechanism and investigate its effect on the infall velocities of the bullet-like systems from the CoDECS (COupled Dark Energy Cosmological Simulations) public database. Five different cDE models are considered: three with constant coupling and exponential potential, one with exponential coupling and exponential potential, and one with constant coupling and supergravity potential. For each model, after identifying the bullet-like systems, we determine the probability density distribution of their infall velocities at the pair separations of $(2-3) R_{\mathrm{vir}}$. Approximating each probability density distribution as a Gaussian, we calculate the cumulative probability of finding a bullet-like system with $V_{c} \geq 3000 \mathrm{~km} / \mathrm{s}$ or $V_{c} \geq 2500 \mathrm{~km} / \mathrm{s}$. Our results show that in all of the five cDE models the cumulative probabilities increase compared to the $\Lambda \mathrm{CDM}$ case and that in the model with exponential coupling $P\left(V_{c} \geq 2500 \mathrm{~km} / \mathrm{s}\right)$ exceeds $10^{-4}$. The physical interpretations and cosmological implications of our results are provided.
\end{abstract}

Subject headings: cosmology:theory — methods:statistical — large-scale structure of universe

\footnotetext{
${ }^{1}$ Astronomy Program, Department of Physics and Astronomy, FPRD, Seoul National University, Seoul 151-747, Korea; jounghun@astro.snu.ac.kr

${ }^{2}$ Excellence Cluster Universe, Boltzmannstr. 2, D-85748 Garching, Germany ; marco.baldi@universecluster.de

${ }^{3}$ University Observatory, Ludwig-Maximillians University Munich, Scheinerstr. 1, D-81679 Munich, Germany
} 


\section{INTRODUCTION}

The splendid success of the standard $\Lambda$ CDM ( $\Lambda$-Cold Dark Matter) cosmology that has been witnessed for the past two decades seems to be overshadowed by the recent discoveries of several possible anomalies (e.g., see Perivolaropoulos 2008, for a review). The so-called "Bullet Cluster" (1E0657-56) is one of those observational challenges that the standard $\Lambda$ CDM cosmology has to face (Mastropietro \& Burkert 2008; Lee \& Komatsu 2010; Thompson \& Nagamine 2011; Akahori \& Yoshikawa 2011). When Tucker et al. (1995) first observed it while looking for a failed cluster, it appeared as a large cloud of hot gas. Later, the Chandra observation revealed that it is in fact a very rare system composed of two headon colliding massive clusters at $z=0.296$ in which a bullet-like subcluster is in the middle of escaping its main cluster's potential well at a bow-shock speed of $\sim 4700 \mathrm{~km} / \mathrm{s}$ after the first core passage (Markevitch et al. 2002, 2004; Clowe et al. 2006; Markevitch 2006).

According to the results of Mastropietro \& Burkert (2008) - based on high-resolution hydrodynamical simulations - the pairwise infall velocity $V_{c}$ of a "bullet" satellite onto the main cluster at a separation of $2 R_{\text {vir }}$ (where $R_{\text {vir }}$ is the virial radius of the main halo) has to exceed $3000 \mathrm{~km} / \mathrm{s}$, or possibly at least $2500 \mathrm{~km} / \mathrm{s}$, in order to reproduce the peculiar morphological properties of the Bullet Cluster, such as the mass ratio between the two colliding clusters, the large separation between the gas and CDM distributions, and the high shock speed inferred from X-ray temperature measurements (see also Springel \& Farrar 2007). In the light of this result, Lee \& Komatsu (2010) investigated how probable it is for a "bullet-like" system to have an infall velocity as high as $3000 \mathrm{~km} / \mathrm{s}$, using the cluster catalogs from the large-volume MICE simulations (Crocce et al. 2010). They found that the probability of finding a bullet-like system with $V_{c} \geq 3000 \mathrm{~km} / \mathrm{s}$ in a WMAP7 universe (Komatsu et al. 2011) is between $\sim 10^{-9}$ and $\sim 10^{-11}$, which led them to conclude that the existence of the Bullet Cluster is incompatible with the $\Lambda$ CDM cosmology. Very recently,

Thompson \& Nagamine (2011) and Akahori \& Yoshikawa (2011) have confirmed the results of Lee \& Komatsu (2010) and of Mastropietro \& Burkert (2008) using the data from different N-body and hydrodynamical simulations, respectively.

Both of a conservative and a radical approach to the Bullet Cluster problem have been recently proposed. The conservative one sought for a lower infall velocity solution in a $\Lambda \mathrm{CDM}$ cosmology under the suspicion that the results from the hydrodynamical simulations or the high shock velocity inferred from the X-ray temperatures may not be trustworthy (e.g., Forero-Romero et al. 2010). In contrast, the radical approach attempted to figure out a mechanism capable of enhancing the infall velocities of the bullet-like systems in nonstandard cosmologies. For example, Wyman \& Khoury (2010) claimed that in models with cascading gravity the probability of a bullet-like system with $V_{c} \geq 3000 \mathrm{~km}$ is four orders of 
magnitude higher than in the $\Lambda$ CDM model (see also Moffat \& Toth 2010). Yet, their result was based on a pure analytical speculation without any numerical backup.

Taking the radical direction, we examine here the possibility that the morphological properties and the required high infall velocity of the Bullet Cluster can be attributed to the presence of coupled Dark Energy (cDE). In cDE models, the Dark Energy is a scalar field $\phi$ with potential $U(\phi)$, which interacts with the CDM particles obeying the following two equations (Wetterich 1995; Amendola 2000, 2004):

$$
\ddot{\phi}+3 H \dot{\phi}+\frac{d U}{d \phi}=\sqrt{\frac{2}{3}} \beta(\phi) \frac{\rho_{c}}{M_{\mathrm{Pl}}}, \quad \dot{\rho}_{c}+3 H \rho_{c}=-\sqrt{\frac{2}{3}} \beta(\phi) \frac{\rho_{c} \dot{\phi}}{M_{\mathrm{Pl}}},
$$

where an overdot represents a derivative with respect to the cosmic time $t, H \equiv \dot{a} / a$ is the Hubble function, $M_{\mathrm{Pl}} \equiv 1 / \sqrt{8 \pi G}$ is the reduced Planck mass, and $\rho_{\mathrm{CDM}}$ is the CDM density. The interaction between the DE scalar field $\phi$ and the CDM fluid determines an exchange of energy-momentum, and a consequent time variation of the CDM particle mass $m_{\mathrm{CDM}}$. In Equations (1) the strength of the coupling is fully determined by the coupling function $\beta(\phi)$, defined as $\beta(\phi) \equiv-d \ln m_{\mathrm{CDM}} / d \phi$.

An enhancement of the infall velocity of the Bullet Cluster is naturally expected in cDE models since a long-range fifth-force generated by the coupling between $\phi$ and CDM particles has been found to play the role of accelerating structure formation processes (e.g., Mangano et al. 2003; Macciò et al.|2004; Mainini \& Bonometto 2006; Pettorino \& Baccigalupi 2008; Baldi et al. 2010; Wintergerst \& Pettorino 2010, and references therein). The level of the enhancement of the infall velocity of the Bullet Cluster will however significantly depend on the shape of the scalar self-interaction potential $U(\phi)$ as well as the coupling function $\beta(\phi)$. We refer to the wide literature on the subject (see e.g., Amendola 2000, 2004; Pettorino \& Baccigalupi 2008; Baldi et al. 2010, 2011; Baldi 2011a, and references therein) for a more thorough description of the main basic features of cDE cosmologies. In particular, the specific models discussed in the present paper have been fully defined and presented by Baldi (2011c) and Baldi (2011d).

The goal of this Paper is to study comprehensively the effect of cDE models on the infall velocities of the bullet-like systems analyzing the public cluster catalogs of the CoDECS project (Baldi 2011d) - the largest suite of cosmological N-body simulations for cDE models to date - which includes various choices for the scalar self interaction potential $U(\phi)$ and the coupling function $\beta(\phi)$. The Paper is organized as follows. In $\S 2$, we provide a brief overview of the N-body simulations for the cDE models and describe how to select the bullet-like systems from the halo catalogs. In $\S 3$, we determine the probability density distribution of the bullet-like systems and calculate the probability of finding a bullet cluster with infall velocity larger than $3000 \mathrm{~km} / \mathrm{s}$ (and $2500 \mathrm{~km} / \mathrm{s}$ as well) for each cDE model. In $\S 4$, we 
provide the physical interpretation of our results and discuss its cosmological implications.

\section{DATA AND ANALYSIS}

\subsection{A Brief Overview of the CoDECS}

The CoDECS (Coupled Dark Energy Cosmological Simulations) project is the largest suite of N-body simulations for $\mathrm{cDE}$ models ever performed, and all its numerical outputs, including halo and sub-halo catalogs, have been recently made publicly available (Baldi 2011d). Using the specific modified version by Baldi et al. (2010) of the widely used parallel Tree-PM N-body code GADGET (Springel 2005), the different runs of the L-CoDECS suite follow the evolution of $1024^{3} \mathrm{CDM}$ and $1024^{3}$ baryonic particles in a periodic cosmological box of linear comoving size $1 h^{-1} \mathrm{Gpc}$ from $z_{i}=99$ to $z=0$ for different realizations of the cDE scenario. The individual CDM and baryonic particles have a mass of $5.84 \times 10^{10} h^{-1} M_{\odot}$ and $1.17 \times 10^{10} h^{-1} M_{\odot}$, respectively, at $z=0$, and the gravitational softening was set at a twenty-fifth of the mean linear interparticle spacing, $\epsilon_{g}=20 h^{-1} \mathrm{kpc}$.

The CoDECS suite includes - besides the standard "fiducial" $\Lambda$ CDM scenario - five different cDE cosmologies: four models with an exponential self-interaction potential (Lucchin \& Matarrese 1985; Ratra \& Peebles 1988; Wetterich 1988)

$$
U(\phi)=A e^{-\alpha \phi},
$$

and one model with a SUGRA potential (Brax \& Martin 1999)

$$
U(\phi)=A \phi^{-\alpha} e^{\phi^{2} / 2},
$$

where for simplicity the scalar field has been redefined in units of the reduced Planck mass $M_{\mathrm{Pl}}$. All the former models have the same value for the potential slope $\alpha=0.08$ but differ from one another in the coupling function: three models (named EXP001, EXP002, and EXP003) are characterized by a constant coupling $\beta(\phi)=$ const. with values $0.05,0.1$, and 0.15 , respectively, consistent with present observational bounds on the DE-CDM interaction (see e.g., Bean et al. 2008; Xia 2009; Baldi \& Viel 2010), while the remaining one (named EXP008e3) has an exponential coupling of the form $\beta(\phi)=0.4 \cdot e^{3 \phi}$. For the latter, instead, a negative constant coupling $\beta=-0.15$ is assumed, allowing for a non-standard evolution of the resulting cDE model as proposed and explained in detail by Baldi (2011c). The combination of a SUGRA potential and of a negative coupling determines a peculiar dynamics of the scalar field, that changes its direction of motion at some intermediate redshift between $z_{\mathrm{CMB}} \approx 1100$ and the present time. As a consequence of this inversion of motion, which for 
the specific model presented here (named SUGRA003) occurs at $z_{\text {inv }} \approx 6.8$, the DE equation of state parameter $w_{\phi}$ shows a "bounce" on the cosmological constant barrier $w_{\phi}=-1$. For this reason this new class of cDE models has been called the "Bouncing cDE scenario" (Baldi 2011c).

All the models included in the CoDECS suite have been normalized according to the latest results from WMAP7 (Komatsu et al. 2011) both for what concerns their background cosmological parameters at $z=0$ and the amplitude of linear density perturbations at the last scattering surface $z_{\mathrm{CMB}} \approx 1100$. The CoDECS runs are therefore fully consistent with present bounds on the perturbations amplitude at $z_{\mathrm{CMB}}$ and allow to investigate the effects that a specific cDE scenario imprints on structure formation processes from $z_{\mathrm{CMB}}$ to the present. Table 1 lists the six cosmological models included in the CoDECS suite, with the corresponding coupling function, potential type, and the expected value of $\sigma_{8}$ that are derived for each scenario with the help of the linear perturbation theory. For a more detailed introduction to the CoDECS project and its related public database, see Baldi (2011d).

It is worth mentioning here that all the cDE models considered here, with the only exception of the SUGRA003 model, could be directly constrained by local measurements of the linear perturbations amplitude $\sigma_{8}$ at low redshifts. In particular, the EXP003 model might be already in tension with presently available constraints on the power spectrum amplitude, $0.78 \leq \sigma_{8} \leq 0.86$, which have been determined by several independent low$z$ observations such as galaxy-galaxy correlation function, cosmic shear statistics, X-ray cluster abundance and Ly- $\alpha$ forest (e.g., McDonald et al. 2005; Hetterscheidt et al. 2007; Henry et al. 2009; Wen et al. 2010, and references therein). On the other hand, the EXP002 and EXP008e3 models appear still marginally consistent with this current limit on $\sigma_{8}$.

\subsection{Selecting the Bullet-Like Systems}

Using the CoDECS public halo catalogs at $z=0$, we first construct a mass-limited $\left(M \geq 10^{13} \mathrm{M}_{\odot} / h\right)$ sample of the cluster-sized halos identified in the simulations of each cosmological model. The CoDECS halo catalogs have been produced by means of a Friends-

of-Friends (FoF) algorithm (Davis et al. 1985) with linking length $\lambda=b \times \bar{d}$, where $\bar{d}$ is the mean inter-particle separation and the linking parameter $b=0.2$ (Baldi 2011d). Reapplying the FoF algorithm with a linking parameter of $b=0.33$ to the mass-limited sample from the CoDECS halo catalogs, we find the clusters-of-clusters for each of which the most massive cluster is identified as the main cluster while the others are considered as satellites, as done in Lee \& Komatsu (2010). 
The bullet-like systems are selected from the identified clusters of clusters according to the same four criteria that were used by Lee \& Komatsu (2010): i) the main cluster's mass $M_{h}$ exceeds a certain cut-off value; $\left.i i\right)$ the main cluster and at least one of its satellites are on the way of head-on collision $(|\cos \alpha| \leq 0.9$, where $\alpha$ is the angle between the velocities of the main cluster and the colliding satellite); iii) the mass ratio between the satellite and main cluster is smaller than one fifth $\left.\left(M_{s} / M_{h} \leq 1 / 5\right) ; i v\right)$ the satellite is located within the distances of $(2-3) R_{\text {vir }}$ from its main cluster where $R_{\text {vir }}$ is the virial radius of its main cluster. The bullet-like systems must satisfy the first three criteria to match the observed properties of the Bullet Cluster (IE0657-57) while the last criterion is used to find the infall pairwise velocities of the main-satellite cluster pairs before the collisions. For the detailed explanation of these criteria, we refer the readers to Lee \& Komatsu (2010).

Regarding the cut-off value of the main cluster's mass, it is worth mentioning here that the main cluster in the observed bullet cluster (IE0657-56) is a very massive one with mass of $M_{h} \geq 10^{15} h^{-1} M_{\odot}$. In the current CoDECS samples, however, there are too few (less than 50) bullet-like systems satisfying $M_{h} \geq 10^{15} h^{-1} M_{\odot}$. To avoid the poor number statistics, we use lower cut-off values, $M_{h} \geq 0.5 \times 10^{15} h^{-1} M_{\odot}$ and $M_{h} \geq 0.7 \times 10^{15} h^{-1} M_{\odot}$. We also focus on the $z=0$ sample for the same reason (at higher redshifts there are too few bullet-like systems).

Table 2 lists the number of clusters $\left(N_{c}\right)$ in the mass-limited sample, the number of the identified clusters of clusters $\left(N_{s c}\right)$, the number and mean pairwise infall velocity of the selected bullet-like systems $\left(N_{\text {bullet }}\right.$ and $\left.\bar{V}_{c}\right)$ for the two different cases of the main cluster's mass cutoff. As can be seen, the cDE models have systematically larger values of $N_{c}, N_{s c}, N_{b u l l e t}$ than the $\Lambda \mathrm{CDM}$ model, which is consistent with the picture that the dark sector coupling leads to a faster growth of the large-scale structures. Regarding the mean infall velocities, all of the cDE models except of the SUGRA003 model yield higher values of $\bar{V}_{c}$. The larger the coupling is, the higher is the mean infall velocity of the bullet-like systems. In the SUGRA003 model, however, the value of $\bar{V}_{c}$ is slightly lower than the $\Lambda$ CDM case when $M_{h} \geq 0.5 \times 10^{15} h^{-1} M_{\odot}$, which should be attributed to the negative value of the coupling constant (see $\S 4$ for more discussions).

\section{INFALL VELOCITIES OF THE BULLET-LIKE SYSTEMS IN cDE MODELS}

For each selected bullet-like system, we measure the relative pairwise velocities, $\mathbf{V}_{c} \equiv$ $\mathbf{V}_{h}-\mathbf{V}_{s}$, where $\mathbf{V}_{h}$ and $\mathbf{V}_{c}$ denote the velocities of the main and satellite clusters, respectively. Then, we bin the values of $\log V_{c}$ and count the number of those bullet-like systems 
with $\log V_{c}$ belonging to a given differential bin, $\left[\log V_{c}, \log V_{c}+d \log V_{c}\right]$, to derive the probability density distribution of $\log V_{c}$ for each cDE model and for the $\Lambda$ CDM model as well, as done in (Lee \& Komatsu 2010).

Figure 1 plots $p\left(\log V_{c}\right)$ at $z=0$ for the case of the standard $\Lambda$ CDM cosmology (solid black histogram) and for the cases of the three cDE models with constant coupling and exponential potential (EXP001, EXP002 and EXP003 as dotted cyan, dashed green and dot-dashed blue histograms, respectively). As can be seen, the distribution $p\left(\log V_{c}\right)$ shows a tendency to develop a longer high-velocity tail in the cDE models with respect to $\Lambda$ CDM. More specifically, the EXP002 model with $\beta=0.1$ exhibits the longest high-velocity tail among the three models.

To quantify the difference among the four distributions and to calculate the cumulative probability of $V_{c}$, we fit $p\left(\log V_{c}\right)$ to a Gaussian distribution by adjusting for each model the mean and standard deviation, $\left(\nu, \sigma_{\nu}\right)$, as done in Lee \& Komatsu (2010). Figure 2 plots the fitting results. As can be seen, the Gaussian distribution gives a reasonably good fit to $p\left(\log V_{c}\right)$ for each case. Table 3 lists the best-fit values of $(\nu, \sigma)$ for the three models, showing that the Gaussian fit to the infall velocity distribution $p\left(\log V_{c}\right)$ has a larger mean and a larger standard deviation in the constant coupling cDE models than in the $\Lambda$ CDM cosmology. The infall velocity distribution with the highest mean is found, as expected, for the case of the EXP003 model, while EXP002 has the largest standard deviation.

Integrating the best-fit Gaussian distributions, we also compute for each model the cumulative probabilities, $P\left(V_{c} \geq 3000 \mathrm{~km} / \mathrm{s}\right)$ and $P\left(V_{c} \geq 2500 \mathrm{~km} / \mathrm{s}\right)$, which are plotted in Figure 3 as a function of the constant coupling $\beta$, where $\Lambda$ CDM corresponds to $\beta=0$. As can be seen, the highest values of the two cumulative probabilities are found for the case of the EXP002 model. The values of $P\left(V_{c} \geq 3000 \mathrm{~km} / \mathrm{s}\right)$ and $P\left(V_{c} \geq 2500 \mathrm{~km} / \mathrm{s}\right)$ increase by a factor of $10^{3}$ and $10^{4}$ in the EXP002 model compared to $\Lambda$ CDM case. It is interesting to note that although the EXP003 model has a stronger coupling, its cumulative probabilities are not higher than those of the EXP002 model.

The same type of analysis has been carried out for the other two cDE models included in the CoDECS suite, namely EXP008e3 and SUGRA003. The results are shown in Figure 4, where the probability density distribution $p\left(\log V_{c}\right)$ computed for these models is plotted and and compared to the $\Lambda$ CDM case, while Figure 5 plots the Gaussian fitting functions whose best-fit means and best-fit standard deviations are listed in Table 3. As can be seen, the probability density distribution $p\left(\log V_{c}\right)$ for the case of the EXP008e3 model has both the largest mean and standard deviation, while the SUGRA003 model yields a smaller mean but a larger standard deviation of $p\left(\log V_{c}\right)$ than $\Lambda$ CDM. 
The cumulative probabilities, $P\left(V_{c} \geq 3000 \mathrm{~km} / \mathrm{s}\right)$ and $P\left(V_{c} \geq 2500 \mathrm{~km} / \mathrm{s}\right)$, are plotted in the top and bottom panels of Figure 6, respectively. As can be seen, both the SUGRA003 and EXP008e 3 models have larger cumulative probabilities than $\Lambda$ CDM. For the case of SUGRA003, when the lower mass limit for the main cluster is set at $0.7 \times 10^{15} \mathrm{M}_{\odot} / h$, the cumulative probabilities, $P\left(V_{c} \geq 3000 \mathrm{~km} / \mathrm{s}\right)$ and $P\left(V_{c} \geq 2500 \mathrm{~km} / \mathrm{s}\right)$, increase by a factor of 10 and $10^{2}$, respectively, compared to $\Lambda$ CDM. However, when the mass cutoff is set at $0.5 \times$ $10^{15} \mathrm{M}_{\odot} / h$, the SUGRA003 model shows almost no enhancement. In contrast, EXP008e3 exhibits the maximal enhancement, no matter which lower mass limit of the main cluster is used. The cumulative probabilities, $P\left(V_{c} \geq 3000 \mathrm{~km} / \mathrm{s}\right)$ and $P\left(V_{c} \geq 2500 \mathrm{~km} / \mathrm{s}\right)$, increase by a factor of $10^{5}$ and $10^{4}$, respectively, compared to the $\Lambda$ CDM result. Note that in this model $P\left(V_{c} \geq 2500 \mathrm{~km} / \mathrm{s}\right)$ reaches up to above $10^{-4}$, which indicates that if the required infall velocity of a bullet-like system can be possibly reduced to $2500 \mathrm{~km} / \mathrm{s}$ (Akahori \& Yoshikawa 2011), then the existence of the Bullet Cluster is not such an extremely rare event in a cDE model like the exponential coupling scenario EXP008e3.

Furthermore, it is worth mentioning here that the above cumulative probabilities are likely to be underestimated due to the limited volume of the CoDECS runs. As shown

in Forero-Romero et al. (2010), the probability of finding a bullet cluster increases almost linearly with the simulation box volume. In other words, the larger volume a simulation box has, the more probable it is to find a bullet-like system with required infall velocity. In fact, we find for the case of $\Lambda$ CDM model $P\left(V_{c} \geq 3000 \mathrm{~km} / \mathrm{s}\right) \sim 10^{-12}$ which is an order of magnitude lower than what Lee \& Komatsu (2010) found, $P\left(V_{c} \geq 3000 \mathrm{~km} / \mathrm{s}\right) \sim 10^{-11}$, from the MICE simulation of volume $27 \mathrm{~h}^{-3} \mathrm{Gpc}^{3}$. Given this, we suspect that the real value of $P\left(V_{c} \geq 3000 \mathrm{~km} / \mathrm{s}\right)$ and $P\left(V_{c} \geq 2500 \mathrm{~km} / \mathrm{s}\right)$ would be higher than our estimates $10^{-6}$ and $10^{-4}$, respectively.

\section{DISCUSSION AND CONCLUSION}

We have carried out a systematic investigation of how the probability of finding a bulletlike system with the required high-infall velocity $(3000 \mathrm{~km} / \mathrm{s}$ or possibly at least $2500 \mathrm{~km} / \mathrm{s}$ ) changes in the context of cDE models compared to the standard $\Lambda \mathrm{CDM}$ cosmology, by analyzing the public numerical data from CoDECS, the largest N-body simulations of cDE models to date, with a box size of 1 comoving $h^{-3} \mathrm{Gpc}^{3}$. Three models with constant coupling and exponential potential (EXP001, EXP002, EXP003), one bouncing cDE model with constant coupling and SUGRA potential (SUGRA003), and one model with exponential coupling and exponential potential (EXP008e3) are considered in the CoDECS suite, besides the fiducial $\Lambda \mathrm{CDM}$ cosmology. 
In all of the five cDE models the cumulative probabilities have been found to be higher than in $\Lambda$ CDM. It has been also shown that the maximal four and five orders of magnitude enhancements of the cumulative probabilities are yielded by the EXP008e3 model where $P\left(V_{c} \geq 2500 \mathrm{~km} / \mathrm{s}\right)$ and $P\left(V_{c} \geq 3000 \mathrm{~km} / \mathrm{s}\right)$ reach up to above $10^{-4}$ and $10^{-6}$, respectively. This is a remarkable result, considering that all the CoDECS simulations share the same random phases in the initial conditions and the same amplitude of density perturbations at the last scattering surface. Furthermore, given the possible underestimate of the cumulative probabilities due to the limited volume of CoDECS (Forero-Romero et al. 2010), we argue that our results should be considered conservative and that therefore the existence of the bullet cluster should not be regarded as an extremely rare event in the EXP008e3 model. Yet, in the other cDE models it should be still quite unlikely to find a bullet cluster with the observed morphology.

The different amplitudes of the bullet velocity enhancement in the different cDE models can be qualitatively understood by considering the time evolution of the linear density and velocity perturbations of each scenario. By taking into account only the coupling strength and its integrated effect on the growth of density perturbations, in fact, our finding that the maximal velocity enhancement is obtained for the exponential coupling model (EXP008e3) might look surprising. As shown by Baldi (2011d) (see the right panel of Figure 2 in the reference), the maximal effect on the density perturbation amplitude is obtained for the EXP003 model that reaches at $z=0$ an enhancement of $\delta(z)$ of about $20 \%$ relative to $\Lambda \mathrm{CDM}$, while the EXP008e3 model shows a 10\% enhancement (as also described by the different $\sigma_{8}$ values listed in Table 1 among the models).

However, if we also consider the velocity perturbation variable $\theta$, defined as

$$
\theta(z) \equiv \frac{1}{\Omega_{c}+\Omega_{b}}\left(\theta_{c} \Omega_{c}+\theta_{b} \Omega_{b}\right)
$$

where $\theta_{b, c} \equiv \vec{\nabla} \cdot \vec{u}_{b, c}$ are the individual velocity perturbation variables, $\vec{u}_{b, c}$ are the peculiar velocities, and $\Omega_{b, c}$ the relative density parameters for baryons and CDM,respectively, we can separately solve for $\theta(z)$ in the different cosmologies, and compare the time evolution of the velocity perturbations to the $\Lambda \mathrm{CDM}$ case. In the linear regime, the density and velocity perturbations $\delta_{b, c}$ and $\theta_{b, c}$ for baryons and CDM in a cDE cosmology evolve according to the following system of coupled differential equations:

$$
\begin{aligned}
\delta_{c}^{\prime} & =\theta_{c}-f\left(\beta_{c}\right) \delta_{c} \\
\theta_{c}^{\prime} & =\left[\frac{1}{2}\left(3 w_{\phi} \Omega_{\phi}+\Omega_{r}-1\right)+g\left(\beta_{c}, \phi^{\prime}\right)\right] \theta_{c}+\frac{3}{2}\left[\Omega_{b} \delta_{b}+\Omega_{c} \delta_{c} \Gamma_{c}\right] \\
\delta_{b}^{\prime} & =\theta_{b}
\end{aligned}
$$




$$
\theta_{b}^{\prime}=\left[\frac{1}{2}\left(3 w_{\phi} \Omega_{\phi}+\Omega_{r}-1\right)\right] \theta_{b}+\frac{3}{2}\left[\Omega_{b} \delta_{b}+\Omega_{c} \delta_{c}\right]
$$

where a prime denotes a derivative with respect to the e-folding time $\alpha \equiv \ln a, \Omega_{\phi}$ and $\Omega_{r}$ are the DE and radiation fractional densities, respectively, $w_{\phi}$ is the DE equation of state parameter, and the three functions $f\left(\beta_{c}\right), g\left(\beta_{c}, \phi^{\prime}\right)$ and $\Gamma_{c}(\phi) \equiv 1+4 \beta_{c}^{2}(\phi) / 3$ encode the deviation from the standard $\Lambda \mathrm{CDM}$ cosmology due to the DE interaction, being $(f, g, \Gamma)=$ $(0,0,1)$ for an uncoupled DE field (see e.g. Amendola 2004, for a more detailed description of the perturbation equations and a definition of the functions $f$ and $g$ ).

By solving the system (5, 5 ) we can separately compute the time evolution of the individual density and velocity perturbations for baryons and CDM and derive the total velocity perturbation $\theta$ given by Equation (4). In Figure 7 we plot the ratio of the velocity perturbation $\theta$ to the $\Lambda \mathrm{CDM}$ case for all the models considered in the present work. As one can see from the figure, the situation is significantly different with respect to the evolution of the density perturbation $\delta$. In particular, we display here for the first time the redshift evolution of the velocity perturbation $\theta$ in a significant number of cDE scenarios, and our results show how a given $\mathrm{cDE}$ model can have a significantly different relative impact on the density and velocity perturbations. Figure 7 clearly shows that the maximal enhancement of velocity perturbations with respect to $\Lambda \mathrm{CDM}$ is realized by the EXP008e 3 model (orange, long-dashed line) - consistently with our findings on the infall velocity in the bullet-like systems identified in the CoDECS simulations - even if the corresponding enhancement of the density perturbations amplitude (i.e. of $\sigma_{8}$ ) is significantly smaller than in the EXP003 model (blue, dot-dashed line).

Although the solution for $\theta$ displayed in Figure 7 is strictly valid only in the linear regime (as for the case of the density perturbation $\delta$ ), the relative impact of the different cDE models on the velocity perturbations can be considered reliable also in the nonlinear regime for situations where the velocity vector is aligned with the gradient of the gravitational potential, since in such a case the extra-friction and the fifth-force contributions arising as a consequence of the DE interaction are also aligned with each other and keep behaving as for the linear case. This is in particular the situation of the bullet-like systems that we are considering in the present work, due to the head-on collision between the two clusters. Therefore we can consider the results obtained for the linear solution of the velocity perturbation $\theta$ as indicative of the impact of the coupling even in these highly nonlinear systems. See Baldi $(2011 b)$ for a discussion on the linear and nonlinear effects of the friction term.

Particularly interesting is also the case of the bouncing cDE model SUGRA003: for this scenario, in fact, the velocity perturbation $\theta$ follows a similar path as for the EXP003 model at high redshifts, but is suddenly slowed down in correspondence to the bounce of the DE 
scalar field at $z_{\mathrm{inv}} \approx 6.8$, and is the only model to have a smaller velocity perturbation $\theta$ as compared to $\Lambda \mathrm{CDM}$ at low redshifts. This is consistent with the background dynamics of the bouncing cDE scenario, that features an inversion of the scalar field motion at $z_{\text {inv }}$ with the consequent change of sign of the friction term $\dot{\phi} \beta(\phi)$, that accelerates particles in their direction of motion before $z_{\text {inv }}$, while it decelerates particles after $z_{\text {inv }}$. The suppression of the velocity perturbation variable $\theta$ after $z_{\text {inv }}$ is therefore an expected effect due to the peculiar dynamics o the bouncing cDE scenario. This is reflected in the lower value of the mean pairwise infall velocity detected in our sample for the Bouncing cDE model (see Table 3), unique among the cDE models considered in our analysis.

Nevertheless, as already mentioned above, the effect of the friction term become more complex in the nonlinear regime, due to the relative orientation between the local velocity field and the gravitational potential gradient. We speculate here that this additional degree of freedom that modulates the efficiency of the friction term in slowing down CDM particle is responsible for the larger variance detected in the pairwise infall velocity distribution in our sample (see again Table 3) which is reflected in a slightly enhanced detection probability even for the SUGRA003 scenario, in spite of the reduction of the mean infall velocity.

To see whether or not the enhanced velocities of the bullet-like systems in cDE models are really related to the enhanced values of the linear velocity perturbations, we compare the ratio of the mean infall velocity of the bullet- like systems for each $\mathrm{cDE}$ model to that for the $\Lambda \mathrm{CDM}$ model, $\bar{V}_{c} / \bar{V}_{\mathrm{c}, \Lambda \mathrm{CDM}}$ (from Table 2) with the values of $\theta / \theta_{\Lambda \mathrm{CDM}}$ at $z=0$ (from Figure 7). The results are plotted in Figures 8 and 9 for the cases of $M_{h} \geq 0.5 \times 10^{15} h^{-1} M_{\odot}$ and $M_{h} \geq 0.7 \times 10^{15} h^{-1} M_{\odot}$, respectively. In each Figure, the dotted line corresponds to the case that the two ratios have the same values. As can be seen, the enhancements of $\bar{V}_{c}$ relative to the $\Lambda$ CDM case indeed follow the pattern of the enhancements of $\theta$ relative to $\theta_{\Lambda \mathrm{CDM}}$. In the SUGRA003 model, however, $\bar{V}_{c} / \bar{V}_{\mathrm{c}, \Lambda \mathrm{CDM}}$ is significantly larger than $\theta / \theta_{\Lambda \mathrm{CDM}}$ at $z=0$, which reflects the peculiar evolution of the SUGRA003 velocity perturbations shown in Figure 7.

To conclude, we have performed a comprehensive investigation of the effect of coupled dark energy models on the pairwise infall velocity of the bullet-like systems identified in the public catalogs of the CoDECS N-body simulations. Since our results have clearly demonstrated that coupled dark energy has the effect of speeding up the bullet-like systems and that the strength of the effect significantly depends on the shape of the scalar potential as well as of the coupling function, we conclude that the Bullet Cluster will become a valuable constraint of the coupled dark energy scenario.

We thank an anonymous referee for useful comments. J.L. acknowledges the financial support from the National Research Foundation of Korea (NRF) grant funded by the Korea 
government (MEST, No.2011-0007819) and from the National Research Foundation of Korea to the Center for Galaxy Evolution Research. M.B. is supported by the DFG Cluster of Excellence "Origin and Structure of the Universe" and by the TRR33 Transregio Collaborative Research Network on the "Dark Universe". 


\section{REFERENCES}

Akahori, T., \& Yoshikawa, K. 2011, arXiv:1109.0826

Amendola, L. 2000, Phys. Rev. D, 62, 043511

Amendola, L. 2004, Phys. Rev. D, 69, 103524

Baldi, M., \& Viel, M. 2010, MNRAS, 409, L89

Baldi, M., Pettorino, V., Robbers, G., \& Springel, V. 2010, MNRAS, 403, 1684

Baldi, M., Lee, J., \& Macciò, A. V. 2011, ApJ, 732, 112

Baldi, M. 2011a, MNRAS, 411, 1077

Baldi, M. 2011b, MNRAS, 414, 116

Baldi, M. 2011c, arXiv:1107.5049

Baldi, M. 2011d, arXiv:1109.5695

Bean, R., Flanagan, É. É., Laszlo, I., \& Trodden, M. 2008, Phys. Rev. D, 78, 123514 Benson, A. J. 2005, MNRAS, 358, 551

Brax, P. H., \& Martin, J. 1999, Physics Letters B, 468, 40

Clowe, D., Gonzalez, A., \& Markevitch, M. 2004, ApJ, 604, 596

Clowe, D., Bradač, M., Gonzalez, A. H., Markevitch, M., Randall, S. W., Jones, C., \& Zaritsky, D. 2006, ApJ, 648, L109

Crocce, M., Fosalba, P., Castander, F. J., \& Gaztanaga, E. 2010, MNRAS, in press, arXiv:0907.0019

Davis, M., Efstathiou, G., Frenk, C. S., \& White, S. D. M. 1985, ApJ, 292, 371

Farrar, G. R., \& Rosen, R. A. 2007, Physical Review Letters, 98, 171302

Forero-Romero, J. E., Gottlöber, S., \& Yepes, G. 2010, ApJ, 725, 598

Henry, J. P., Evrard, A. E., Hoekstra, H., Babul, A., \& Mahdavi, A. 2009, ApJ, 691, 1307

Hetterscheidt, M., Simon, P., Schirmer, M., et al. 2007, A\&A, 468, 859

Komatsu, E., et al. 2011, ApJS, 192, 18 
Lee, J., \& Komatsu, E. 2010, ApJ, 718, 60

Lucchin, F., \& Matarrese, S. 1985, Phys. Rev. D, 32, 1316

Macciò, A. V., Quercellini, C., Mainini, R., Amendola, L., \& Bonometto, S. A. 2004, Phys. Rev. D, 69, 123516

Mainini, R., \& Bonometto, S. 2006, Phys. Rev. D, 74, 043504

Mangano, G., Miele, G., \& Pettorino, V. 2003, Modern Physics Letters A, 18, 831

Markevitch, M. 2006, in Proc. X-Ray Universe 2005, ed. A Wilson (ESA SP-604; Noordwijk: ESA), 723

Markevitch, M., Gonzalez, A. H., David, L., Vikhlinin, A., Murray, S., Forman, W., Jones, C., \& Tucker, W. 2002, ApJ, 567, L27

Markevitch, M., Gonzalez, A. H., Clowe, D., Vikhlinin, A., Forman, W., Jones, C., Murray, S., \& Tucker, W. 2004, ApJ, 606, 819

Markevitch, M., Govoni, F., Brunetti, G., \& Jerius, D. 2005, ApJ, 627, 733

Mastropietro, C., \& Burkert, A. 2008, MNRAS, 389, 967

McDonald, P., Seljak, U., Cen, R., et al. 2005, ApJ, 635, 761

Moffat, J. W., \& Toth, V. T. 2010, arXiv:1005.2685

Nusser, A. 2008, MNRAS, 384, 343

Perivolaropoulos, L. 2008, arXiv:0811.4684

Pettorino, V., \& Baccigalupi, C. 2008, Phys. Rev. D, 77, 103003

Ratra, B., \& Peebles, P. J. E. 1988, Phys. Rev. D, 37, 3406

Springel, V. 2005, MNRAS, 364, 1105

Springel, V., \& Farrar, G. R. 2007, MNRAS, 380, 911

Thompson, R., \& Nagamine, K. 2011, arXiv:1107.4645

Tucker, W. H., Tananbaum, H., \& Remillard, R. A. 1995, ApJ, 444, 532

Wen, Z. L., Han, J. L., \& Liu, F. S. 2010, MNRAS, 407, 533 
Wetterich, C. 1988, Nuclear Physics B, 302, 668

Wetterich, C. 1995, A\&A, 301, 321

Wintergerst, N., \& Pettorino, V. 2010, Phys. Rev. D, 82, 103516

Wyman, M., \& Khoury, J. 2010, Phys. Rev. D, 82, 044032

Xia, J.-Q. 2009, Phys. Rev. D, 80, 103514 


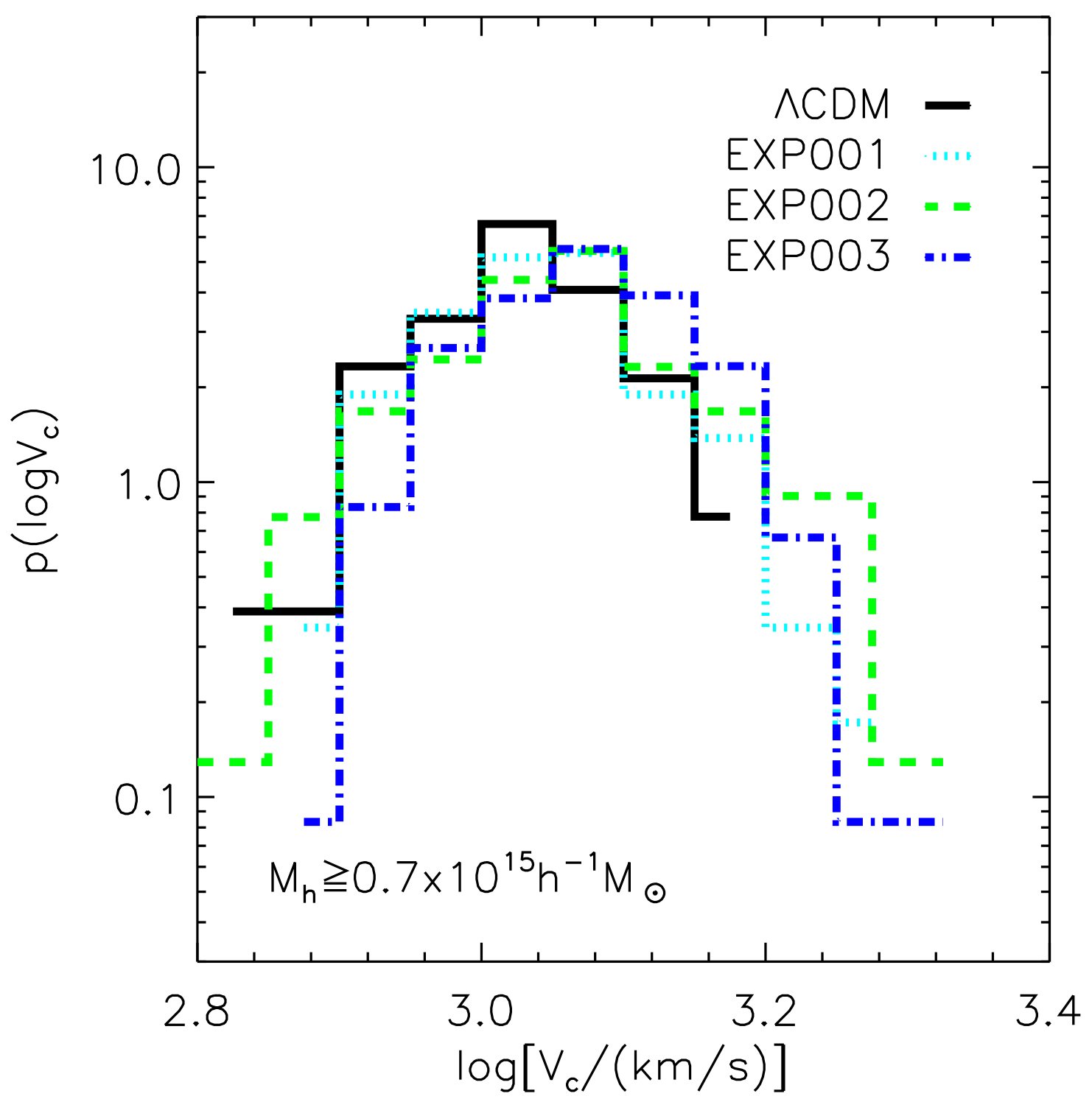

Fig. 1.- Probability density distribution of the infall velocities, $\log V_{c}$, of the bullet-clusterlike systems measured within $2 \leq r / R_{200} \leq 3$ at $z=0$ for the four different dark energy models: $\Lambda$ CDM, EXP001, EXP002, and EXP003 as dashed, dotted, solid and dot-dashed histograms, respectively. The main cluster masses are $M_{\text {main }} \geq 0.7 \times 10^{15} h^{-1} M_{\odot}$, for each case. 


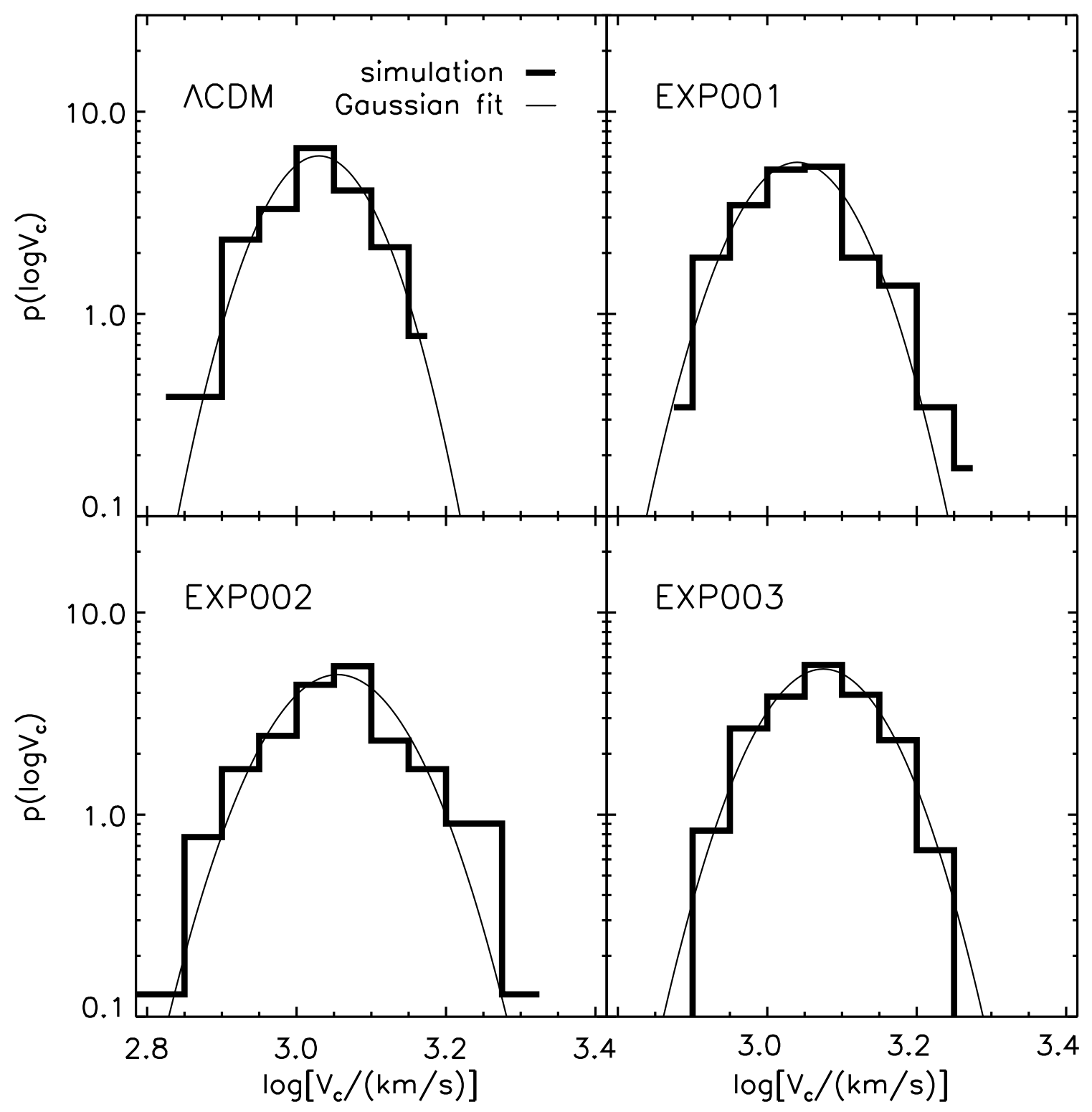

Fig. 2.- Gaussian-fit (thin solid line) to the probability density distribution of the infall velocities, $\log V_{c}$, of the bullet-cluster-like systems measured within $2 \leq r / R_{200} \leq 3$ at $z=0$ (thick solid histogram) for the four different dark energy models: $\Lambda$ CDM, EXP001, EXP002, and EXP003 in the top-left, top-right, bottom-left and bottom-right panel, respectively. The main cluster masses are $M_{\text {main }} \geq 0.7 \times 10^{15} h^{-1} M_{\odot}$, for each case. 


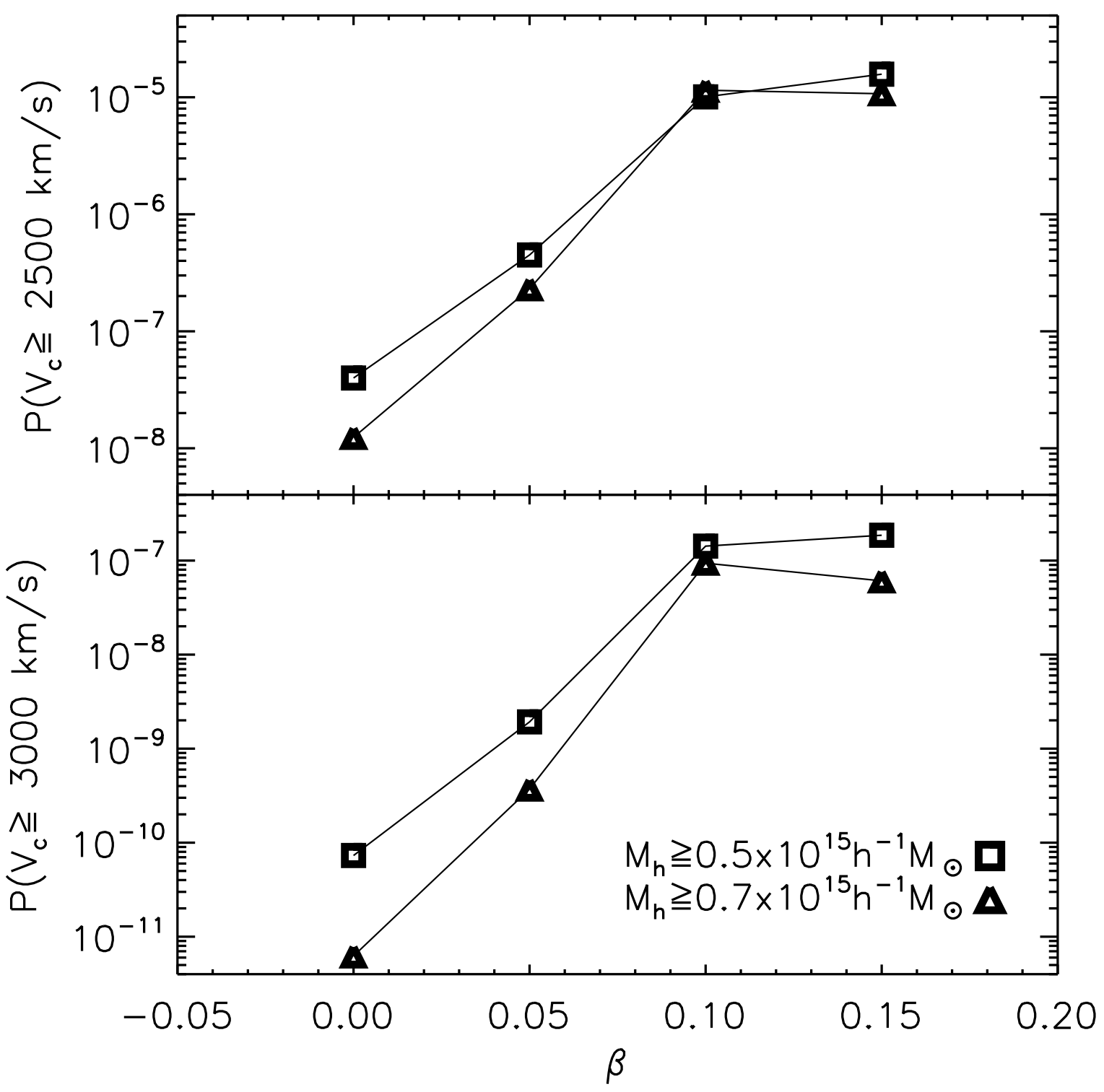

Fig. 3.- Cumulative probabilities of the infall velocities of the bullet clusters versus the cDE coupling constant, $\beta$ for the $\Lambda$ CDM, EXP001, EXPOO2, and EXP003 models. 


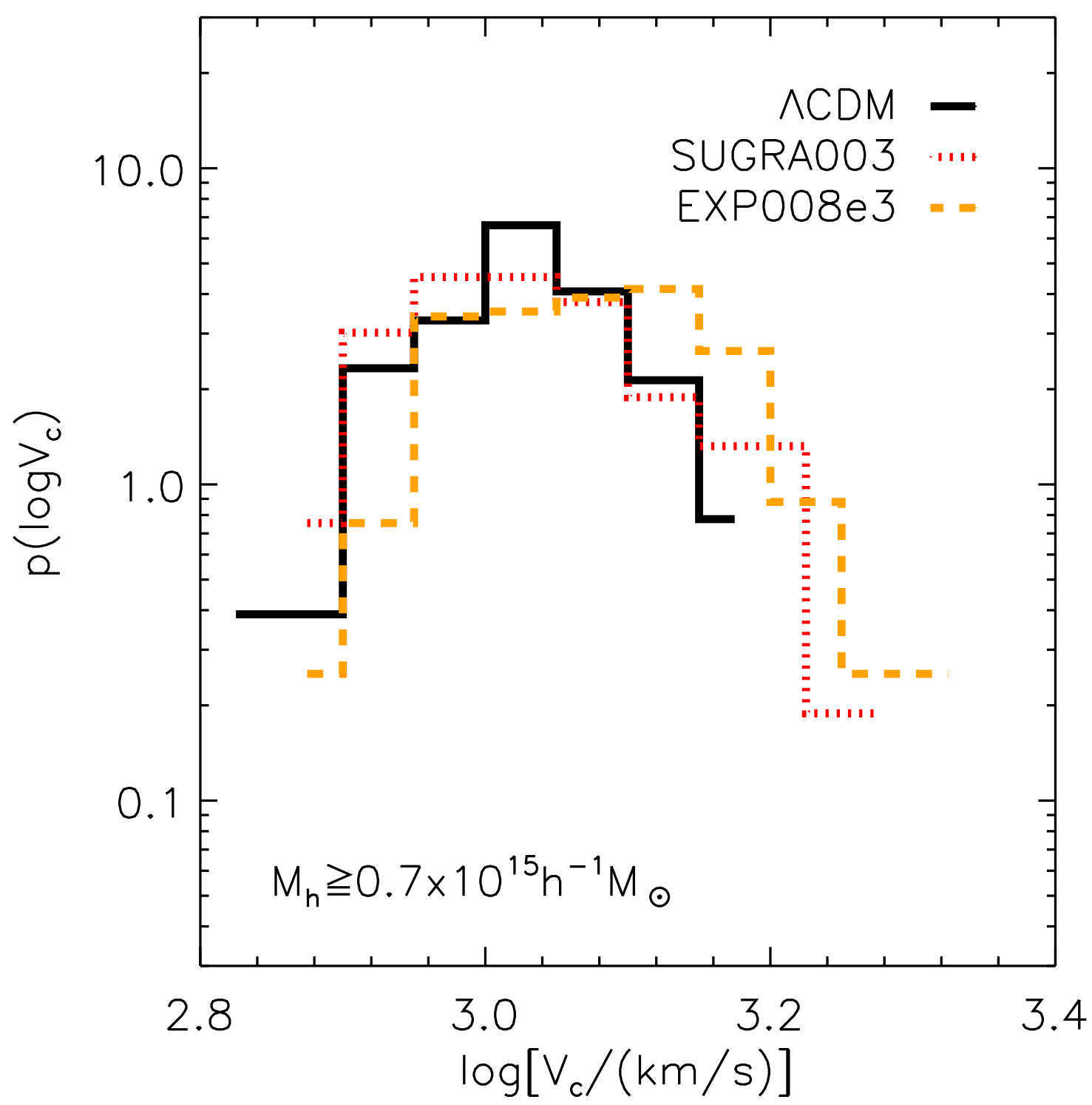

Fig. 4. - Same as Figure 1 but for the SUGRA003 and EXP008e3 models (dotted and dashed histograms, respectively) in comparison with the $\Lambda$ CDM case (solid histogram). 


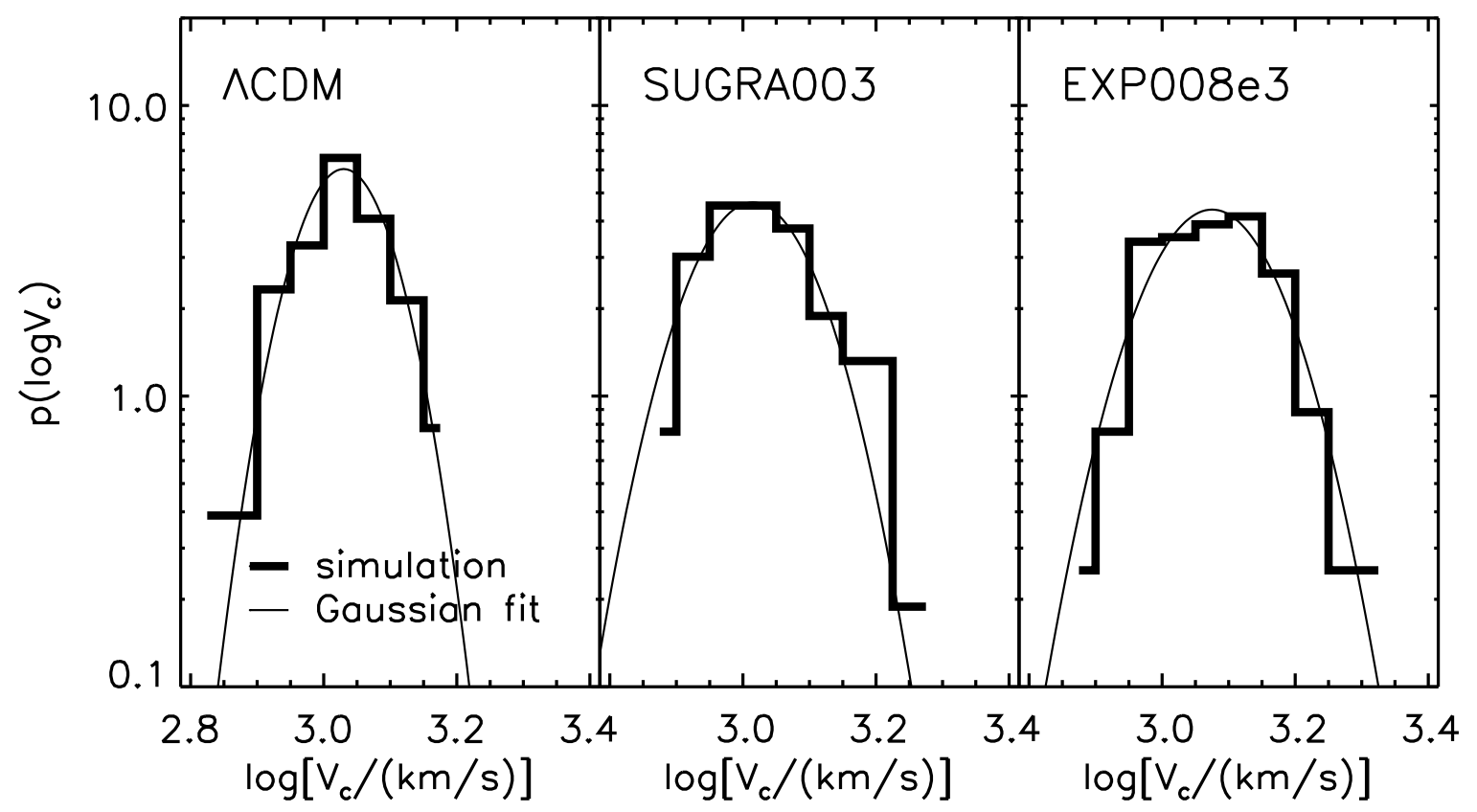

Fig. 5.- Same as Figure 2 but for the SUGRA003 and EXP008e3 models in comparison with the $\Lambda$ CDM case. 


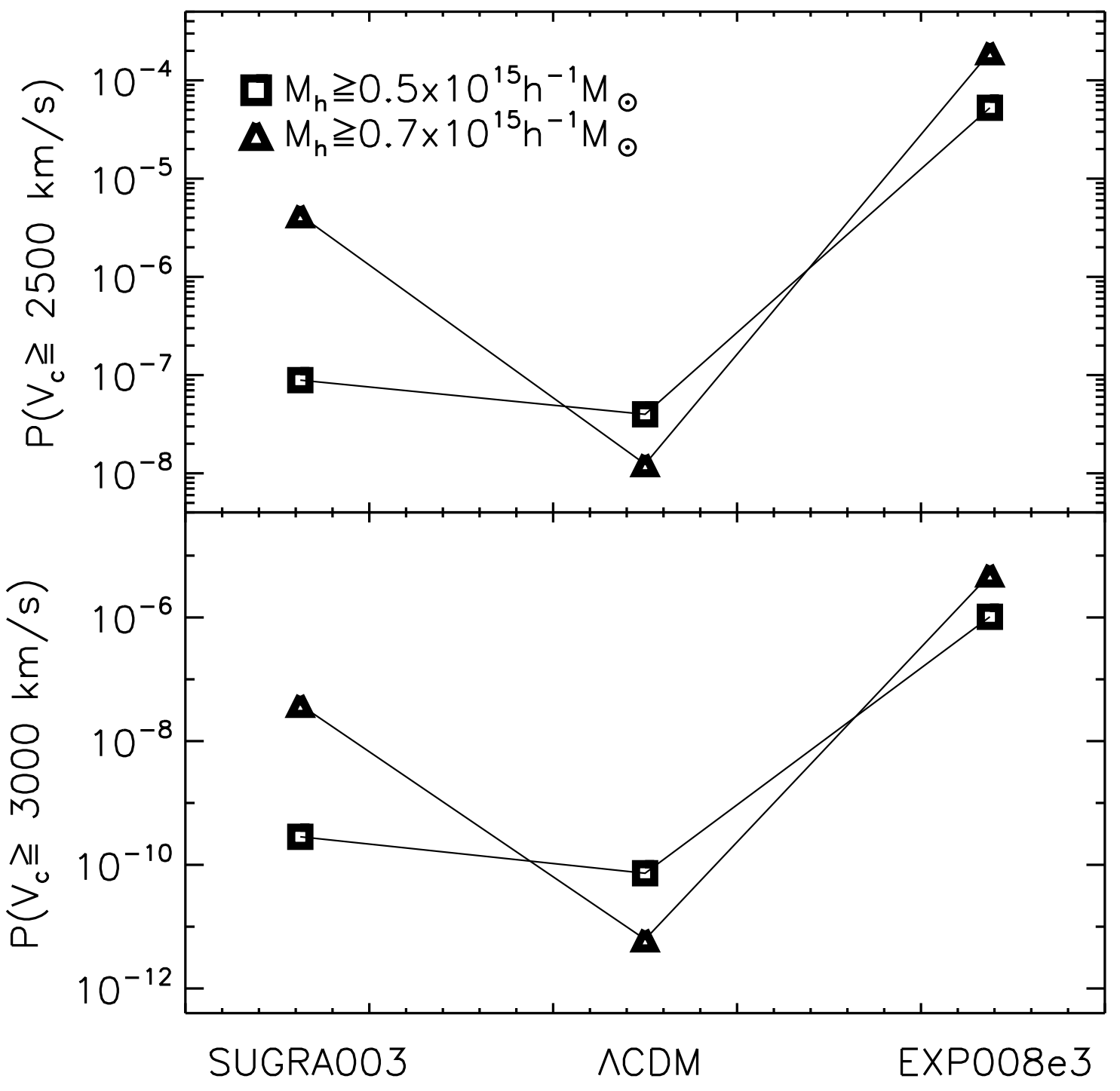

Fig. 6.- Cumulative probabilities of the infall velocities of the bullet clusters versus the cDE coupling constant, $\beta$ for the SUGRA003 AND EXP008e3 models. 


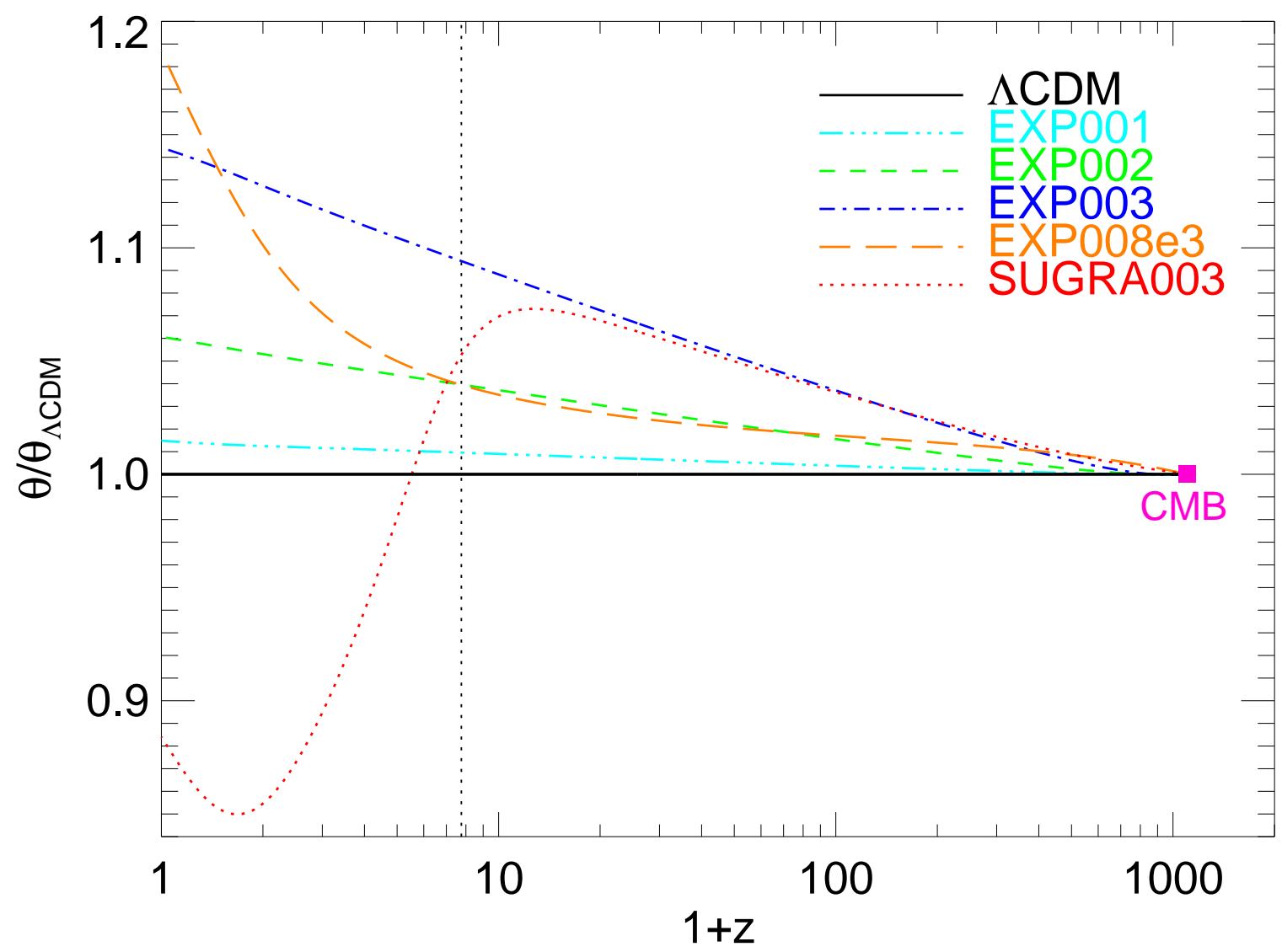

Fig. 7.- Velocity perturbation $\theta$ for all the cDE models considered in the present work relative to the $\Lambda \mathrm{CDM}$ case $\theta_{\Lambda \mathrm{CDM}}$. The vertical dotted line indicates the redshift at which the Bouncing cDE model SUGRA003 features the inversion of the scalar field motion, corresponding to $z_{\text {inv }} \approx 6.8$. Note that when this transition occurs at $z_{\text {inv }}$ the trend of the linear velocity perturbation of the SUGRA003 model with respect to $\Lambda$ CDM case is also inverted. On the other hand the fast growth of the coupling function at low redshifts for the EXP008e3 model leads to the steep increase in the velocity perturbation relative to the $\Lambda \mathrm{CDM}$ case. In consequence the EXP008e 3 model has a larger value of $\theta / \theta_{\Lambda \mathrm{CDM}}$ at $z=0$ than the EXP003 model even though the overall amplitude of the linear density perturbation is higher in the EXP003 model. 


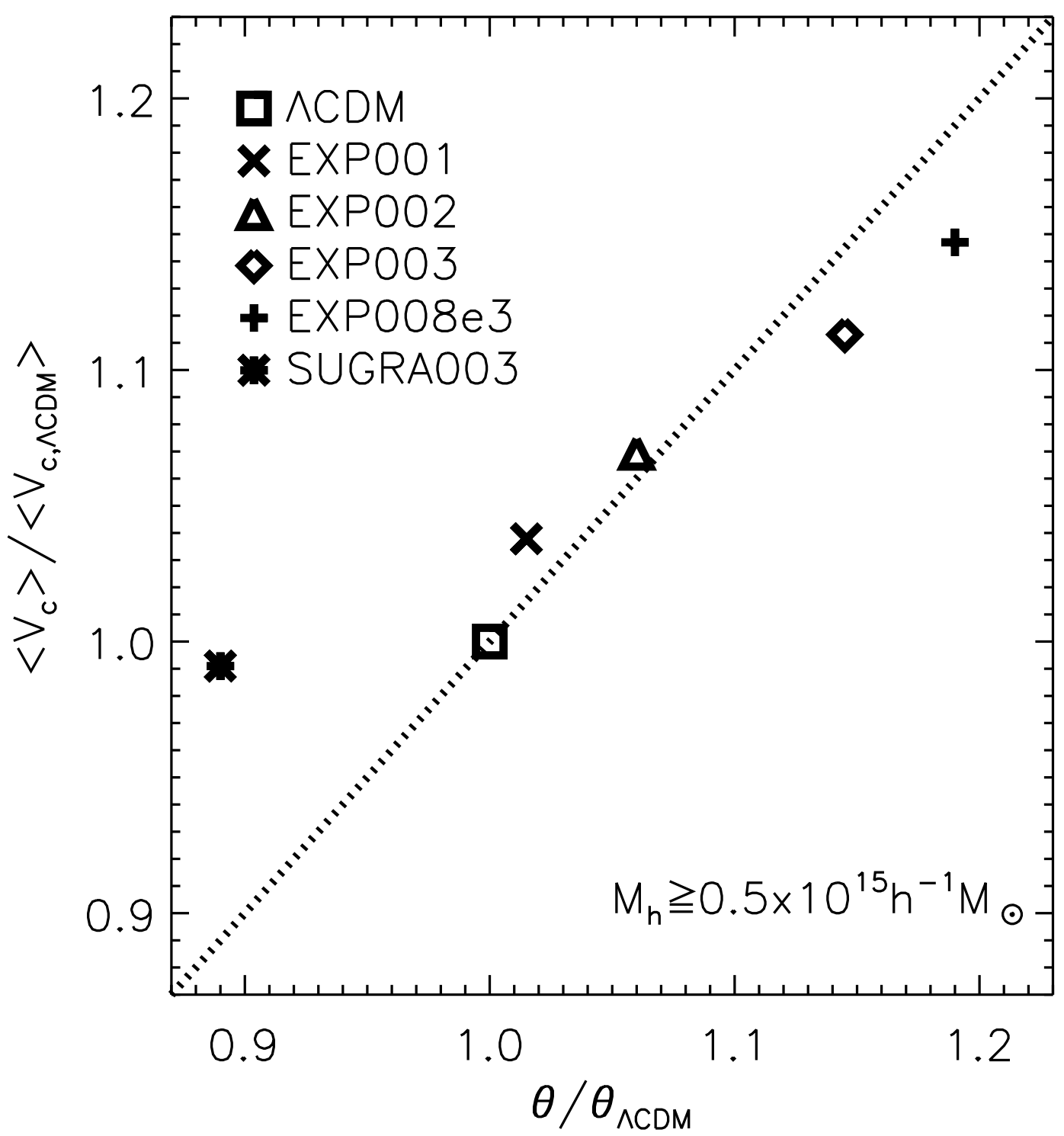

Fig. 8. - Ratio of the mean infall velocities for all the models to that for the $\Lambda$ CDM case versus the theoretical predicted ratio of the velocity perturbations for all models to that for the $\Lambda \mathrm{CDM}$ case. The mean infall velocities are obtained by taking the averages over the selected bullet-like systems with $M_{h} \geq 0.5 \times 10^{15} h^{-1} M_{\odot}$. The dotted line corresponds to the case of $\left\langle V_{c}\right\rangle /\left\langle V_{\mathrm{c}, \Lambda \mathrm{CDM}}\right\rangle=\theta / \theta_{\Lambda \mathrm{CDM}}$. 


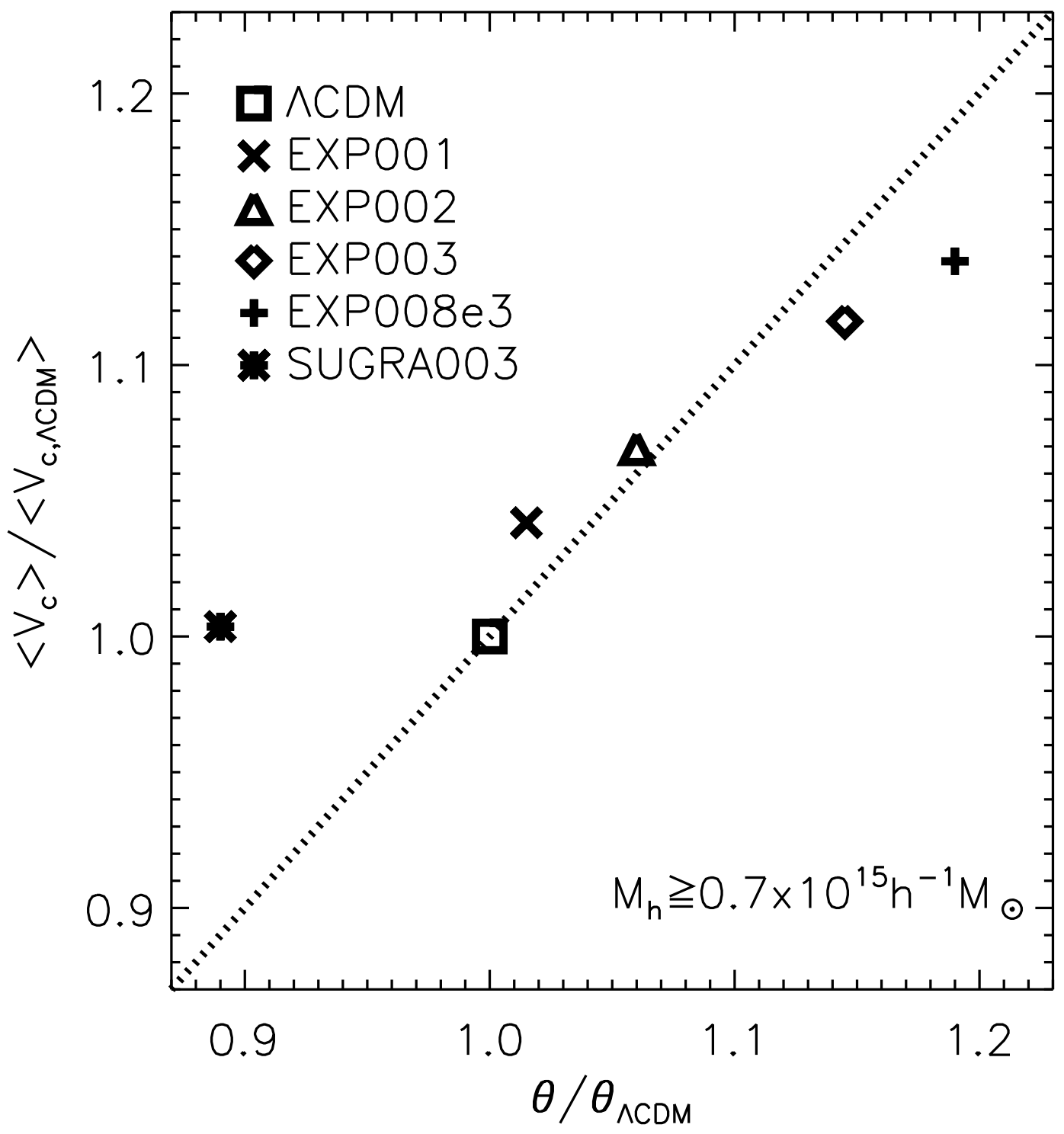

Fig. 9. - Same as Figure 8 but for the case of $M_{h} \geq 0.7 \times 10^{15} h^{-1} M_{\odot}$. 
Table 1. Defining properties of the six cosmological models (see Baldi 2011d, for the detailed descriptions of the models).

\begin{tabular}{ccccc}
\hline \hline Model & $U(\phi)$ & $\alpha$ & $\beta(\phi)$ & $\sigma_{8}$ \\
\hline$\Lambda$ CDM & const. & - & - & 0.809 \\
EXP001 & $e^{-\alpha \phi}$ & 0.08 & 0.05 & 0.825 \\
EXP002 & $e^{-\alpha \phi}$ & 0.08 & 0.1 & 0.875 \\
EXP003 & $e^{-\alpha \phi}$ & 0.08 & 0.15 & 0.967 \\
EXP008e3 & $e^{-\alpha \phi}$ & 0.08 & $0.4 e^{3 \phi}$ & 0.895 \\
SUGRA003 & $\phi^{-\alpha} e^{\phi^{2} / 2}$ & 2.15 & -0.15 & 0.806 \\
\hline
\end{tabular}


Table 2. Model, number of clusters, number of clusters of clusters, number and mean infall velocity of bullet-like systems for the two different cases of the main cluster masses

\begin{tabular}{ccccccc}
\hline \hline model & $N_{c}$ & $N_{s c}$ & $N_{\text {bullet }}{ }^{\mathrm{a}}$ & $N_{\text {bullet }}{ }^{\mathrm{b}}$ & $\begin{array}{c}\bar{V}_{c}^{\mathrm{a}} \\
{[\mathrm{km} / \mathrm{s}]}\end{array}$ & $\begin{array}{c}\bar{V}_{c}^{\mathrm{b}} \\
{[\mathrm{km} / \mathrm{s}]}\end{array}$ \\
\hline$\Lambda$ CDM & 121936 & 22019 & 184 & 103 & 999.56 & 1073.33 \\
EXP001 & 124467 & 22448 & 195 & 116 & 1037.38 & 1118.27 \\
EXP002 & 131880 & 23648 & 231 & 155 & 1069.03 & 1247.71 \\
EXP003 & 141915 & 25223 & 357 & 240 & 1112.55 & 1197.93 \\
EXP008e3 & 132992 & 23795 & 233 & 159 & 1146.49 & 1221.66 \\
SUGRA003 & 127651 & 23216 & 188 & 106 & 990.53 & 1077.21 \\
\hline
\end{tabular}

${ }^{\text {a For }} M_{h} \geq 0.5 \times 10^{15} h^{-1} M_{\odot}$.

${ }^{\mathrm{b}}$ For $M_{h} \geq 0.7 \times 10^{15} h^{-1} M_{\odot}$. 
Table 3. Model and corresponding best-fit values of the mean and standard deviation of the Gaussian fitting function

\begin{tabular}{ccc}
\hline \hline model & $\left(\nu, \sigma_{\nu}\right)^{\mathrm{a}}$ & $\left(\nu, \sigma_{\nu}\right)^{\mathrm{b}}$ \\
\hline$\Lambda$ CDM & $(2.99,0.076)$ & $(3.03,0.066)$ \\
EXP001 & $(3.00,0.081)$ & $(3.04,0.071)$ \\
EXP002 & $(3.01,0.091)$ & $(3.06,0.081)$ \\
EXP003 & $(3.04,0.086)$ & $(3.08,0.076)$ \\
EXP008e3 & $(3.05,0.091)$ & $(3.08,0.091)$ \\
SUGRA003 & $(2.98,0.081)$ & $(3.02,0.086)$ \\
\hline
\end{tabular}

${ }^{\text {a For }} M_{h} \geq 0.5 \times 10^{15} h^{-1} M_{\odot}$.

${ }^{\mathrm{b}}$ For $M_{h} \geq 0.7 \times 10^{15} h^{-1} M_{\odot}$. 